\title{
ASSOCIAÇÃO ENTRE SAI INOMICINA E TIAMULIN EM RAÇÕES DE AVES PARA CORTE
}

\author{
EFFECT OF SALINOMYCIN AND TIAMUIJIN ASSOCIATION \\ IN BROILER CHICKEN DIETS
}

Ricardo de AI,BLQLERQUEE '; Esleibe (;HION ${ }^{2}$; Cesar Gonçalves de LIMA'; Antônio Froilano Mello de CARVALHO ${ }^{3}$; Aldair Marques de CaRVAL,HO ${ }^{4}$; Antônio (Guilherme Machado de CASTRO t; Luiz Cesar BEILACAAMBA

\begin{abstract}
RESUMO
A pesquisa foi conduzida no Aviário Experimental do Departamento de Produção Animal da Faculdade de Medicina Veterinária e Zootecnia da Universidade de São Paulo, em Pirassununga. São Paulo, e teve a finalidade de verificar a possível toxicidade do anticoccidiano salinomicina pela administração simultânea do antibiótico tiamulin na ração. Foram utilizados 1.296 pintos de 01 dia. distrihuídos em 36 boxes experimentais e submetidos a 6 tratamentos, com 6 repetiçōes de 36 aves cada. O delineamento experimental foi o de blocos casualizados. Para os tratamentos foram empregados $60 \mathrm{ppm}$ de salinomicina e 0 ; 10: 1.5: 20: 30 e $40 \mathrm{ppm}$ de tiamulin. Os dados oblidos indicaram não haver depressão no crescimento das aves. nem tampouco no consumo de ração e conversão alimentar. Não ocorreram lesões nos sacos aéreos e os testes de soroaglutinação rápidal para Mycoplasma gallissepticum apresentaram resultados negativos. Tendo em vista os resultados obtidos, pode-se dizer que a adição simultânea de tiamulin e salinomicina em rações de aves para corte. nos níveis utilizados, não acarreta toxicidade.
\end{abstract}

UNITERMOS: Nutrição: Envenenamento: Tiamulin; Salinomicina; Frangos de corte; Antibióticos

\section{INTRODUÇÃO}

Entre os vários aditivos às rações das aves, o tiamulin é um antibiótico semi-sintético com pronunciada atividade contra vários organismos gram-positivos e espécics de Mycoplasma, sendo usado na criação de frangos para corte. para tratar as infeç̧ões ou prevenir os prejuízos econômicos delas decorrentes.

No grupo de antibióticos ionóforos que são amplamente usados na produção avícola como coccidiostáticos, e também como reguladores da fermentação ruminal em gado de corte. encontram-se além da salinomicina, a monensina, a narasina e a lasalocida.

Há inúmeros relatos na literatura, concernentes a níveis efetivos de salinomicina capazes de controlar a coccidiose em aves (DANFORTH et al.2.3, 1977; MIGAKI el al.9, 1979; MORRISON et al.10, 1979; YVORE et al.15, 198()), sendo escassas as informações relativas à sua toxicidade.

Investigações de DANFORTH et al.2.3 (1977) mostraram que a salinomicina, nas dosagens de 60 a $100 \mathrm{ppm}$, não apresentou toxicidade em aves mantidas tanto em piso quan- to em baterias, enquanto MIGAKI ct al. ${ }^{9}$ (1979) observaram uma leve depressão significativa no crescimento de aves criadas em piso, quando em sua dieta foram acrescentadas. respectivamente, $80 \mathrm{ppm}$ e 100 a $160 \mathrm{ppm}$ daquela droga. Por sua vez, e no mesmo sistema de criação. YVORE et al.15 (1980) observaram significativa diminuição no crescimento, usando $80 \mathrm{ppm}$ de salinomicina, enquanto MORRISON et al. 10 (1979) evidenciaram apenas um leve prejuízo.

Sendo os ionóforos freqüentemente usados em avicultura. indagações sobre suas possíveis interaçōes com outras drogas têm sido levantadas. Estas interaçōes geralmente são indistinguíveis da intoxicação causada somente por ionóforos, principalmente quanto aos sintomas clínicos e alterações histopatológicas. Em aves, a anorexia, perda de peso e miastenia aparecem como sintomas, observando-se miopatia em investigações histopatológicas (SCZANCER 13, 1989).

Descriçōes de interaçōes tóxicas entre monensina e tiamulin sāo apresentadas por FRIGG4 (1979) e WEISMAN I4 (1980), os quais informaram que a administração simultânea 
de ambat determinou severa imtoxicalgaio aguda. com altat morlalidiale.

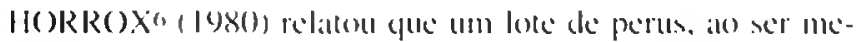
dicado com tiamulin. apresenton mortablidade de aproxi-

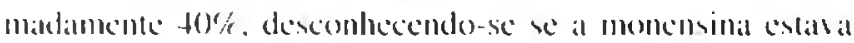

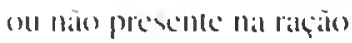

A mancira pela cual a interaça contre momemsina c liamulin

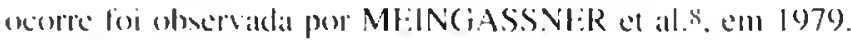
() pesquisadores rebataram. em estudas de perfusão com

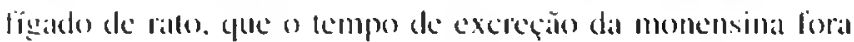
atumentado em ofo\% pelat addiçäo de tiamulin. e que os eleitos

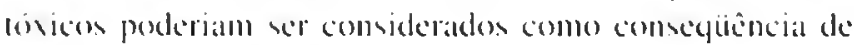
niveis circulatorion allumentados do ionsiforo. calluadon por

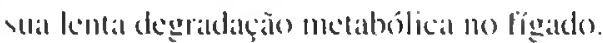

An estudatren o use simultâneo de sillinomicina c tiamulin

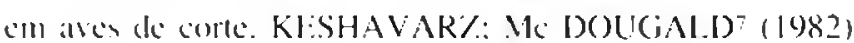
evidenciatran que alo nivel recomendido de inclusão (60)

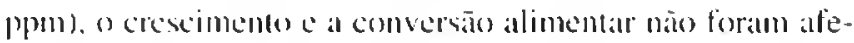

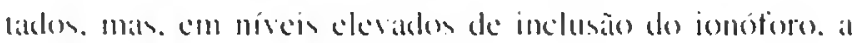
produtividade for prejudicada e os eleitos adversos

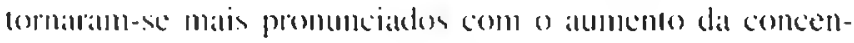
traçüo Ma dicta.

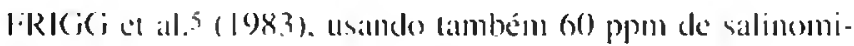

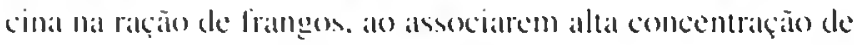
tiamulin (250 ppm) nal agua cke hebida. durante 3 dias. entre

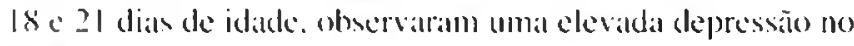
crescimento.

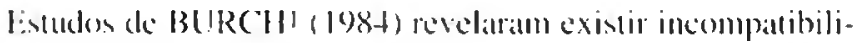
dade entre on ionóloren monemsinat. salinomicina e nanasina. com tiamulin. () atutor alfirma ser a mesma relateionada a

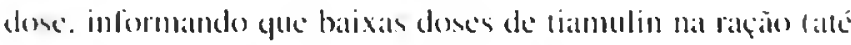

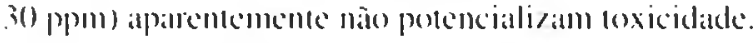

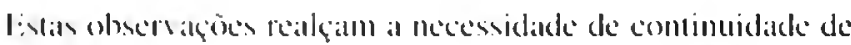
cotuder sobre a compattibilidade mútua de drogas corrente-

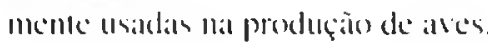

() presente lababalho foi condurido com o ohjelivo de erilicar a losicidade de salinomicina em ares pallal corte. pela

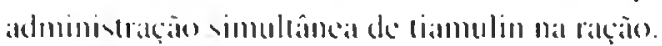

\section{MATKRIAI E METOHOO}

() experimemto loi realisado no Aviario beperimental do Departamento de Produçán Animal dal Faculdade de Medicina Velerinária e Yonteconia dal I'niversidade de Säo Paulo. locallisado em Pirimununga - SP. no periodolo de $28 / 11 / 89$ a $16 / 01 / 90$. comn duraçión de +9 diats.
Foram utilizados 1.296 pintos de um dial para cortce sexados. da linhagem comercial Ross. on quais foram alojaklos em um galpano experimental dividido em 30 boxes (de 1.70 x 2.50

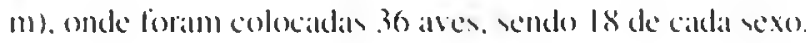

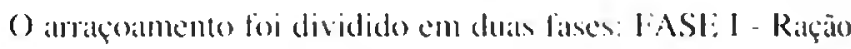
pré-incial (de I a II dias) e Ralçio incial (de 12 a 2.3 dias):

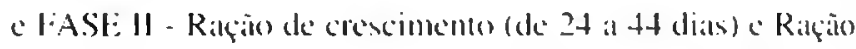
de retirada de drogas on lerminaçio (de 4.5 a th) dias).

As rações foram formuladas bilseando-se no NR('1 (1984). para allender as exigências nutricionais das ares. Listas

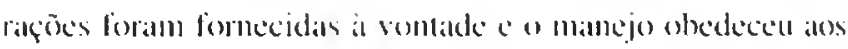
padroon ustais parat a criaçäo de liangon de corte.

() delineamento experimental foi em hlocos casualizados. constituído por 6 matamentos com 6 repetiçoes. As análises de variância foram feitas de acordo com SNEDI:COR: COCHRAN'12 (1980). milizando-se oleste de I)unnell parat a comparaçăo entre an médias.

Os tratamentos constaram da suplementaçăo de raçós lestes com tiamulin e salinomicina (Tab. I ). mas diferemtes fases de

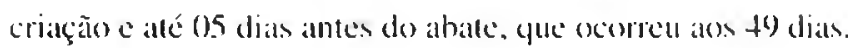

YAKI:A

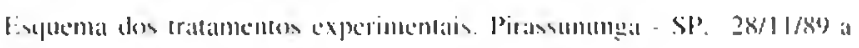
$16(01 / 4)$

\begin{tabular}{|c|c|c|}
\hline Triltallmentus & $\begin{array}{l}\text { Ji:mulin } \\
\text { ppun }\end{array}$ & $\begin{array}{c}\text { Sillimommanal } \\
\text { гриน }\end{array}$ \\
\hline$\therefore$ & (11) & $(x)$ \\
\hline 13 & 19 & (n) \\
\hline$c^{\prime}$ & 20 & 60 \\
\hline 1) & i) & (1) \\
\hline $1:$ & 411 & (10) \\
\hline 1 & & (n) \\
\hline
\end{tabular}

Vo 1". 35". 45" dias de idade das ales. anmostras de sangue de 18 alder por tratamento (3 ares por parcelat) foram colthidas e lestadas sorologicamente para Mucophasma sp. antases de SAR (Soro Aglutinatçito Rápida). exame este realisaldo no) L.aboratório de Pallologia Avícolal do Instituto Biológico. em Descallato - SP. 


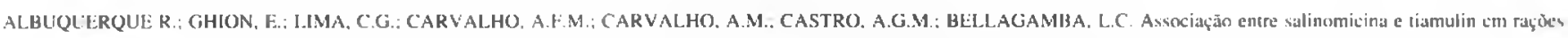
de anes para conte. Brax. J. vet. Res. anim. Sci.. São Paulo. v.32. n..3, p. 165-169. 1995

Aos 45 dias de idade foram retiradas, ao acaso, 6 aves por tratamento, uma ave de cada box, e as mesmas foram necropsiadas e submetidas a uma avaliação (escore) macro e microscópica de lesôes.

As variáveis consumo de ração, conversão alimentar. peso médio, ganho médio diário e mortalidade foram registradas e analisadas aos 23 e 49 dias.

\section{RESULTADOS E DISCUSSÃO}

Os dados da Táb. 2, demonstram que não houve diferenças significativas $(P<0,05)$ na comparação do ganho em peso das aves alimentadas com as raçóes contendo salinomicina e diferentes niveis de liamulin, indicando que a utilização simultânea destes dois componentes, nas dosagens estudadas. não deprime o ganho em peso normal das aves. Estes achados estão de acordo com os resultados obtidos por DANFORTH et al.2.3 (1977).

\section{TABFI,A 2}

Peso corporal médio e ganho médio diário das aves. Pirassununga - SP. $28 / 11 / 89$ a $16 / 01 / 90$.

\begin{tabular}{cccc}
\hline Tratamentos & $\begin{array}{l}\text { Peso corporal } \\
23 \text { dias }\end{array}$ & $\begin{array}{l}\text { (g/ave) } \\
49 \text { dias }\end{array}$ & $\begin{array}{c}\text { Gantho médio diário (g) } \\
\text { I }-49 \text { dias }\end{array}$ \\
\hline A & 787.56 & 2257.12 & 45.16 \\
B & 804.9 .5 & 2297.10 & 46.01 \\
C & 798.68 & 2257.30 & 45.17 \\
D & 806.32 & 2229.68 & 44.62 \\
F & 788.65 & 2225.31 & 44.55 \\
F & 801.10 & 2218.10 & 44.35 \\
\hline
\end{tabular}

Considerando-se os valores de consumo de ração (Tab. 3), não foi observada diferença significativa entre os tratamentos que incluíam tiamulin. quando comparados com as raçōes sem sua presença. A adição de tiamulin nas rações, tanto na presença quanto na ausência de salinomicina, não afetou o consumo de ração das aves.

Com relação à conversão alimentar (Tab. 3), observa-se que a ausência de tiamulin induziu um pior desempenho, embora não fosse detectada diferença significativa entre os resultados obtidos. KESHAVARZ; Mc DOUGALD7 (1982), ao trabalharem com igual concentração de salinomicina, também não observaram alteração quanto a crescimento e conversão alimentar, enquanto FRIGG et al.5 (1983) ao associarem ao ionóforo uma alta concentração de tiamulin, regis- traram grande depressão no crescimento. Esta ocorrência poderia ser explicada, segundo BURCH' (1984), pelo fato de o tiamulin, em alta concentração, poder impedir o sistema de excreção do ionóforo.

\section{TABELA 3}

Medias de consumo de ração e conversão alimentar. Pirassununga - SP. $28 / 11 / 89$ a $16 /(11 / 90)$.

\begin{tabular}{|c|c|c|c|c|}
\hline \multirow[t]{2}{*}{ Tratamentos } & \multicolumn{2}{|c|}{ Consumo de ração (g/ave) } & \multirow{2}{*}{$\begin{array}{l}\text { Conversão } \\
\text { l-2.3 diass }\end{array}$} & \multirow{2}{*}{$\begin{array}{l}\text { Alimentar } \\
1-49 \text { dias }\end{array}$} \\
\hline & 1-23 dias & 1.49 dias & & \\
\hline A & 1152.9 & 424.3 .4 & 1.45 & 1.92 \\
\hline B & 1172.8 & +425.7 & 1.45 & 1.92 \\
\hline $\mathrm{C}$ & 1162.6 & $+3.51,7$ & 1.44 & 1.92 \\
\hline D & 1161.7 & 4253.0 & 1.43 & 1.90 \\
\hline E & 1166.3 & 4322.7 & 1,47 & 1.9 .3 \\
\hline $\mathrm{F}$ & 1164.8 & 4332.4 & 1.45 & 1.95 \\
\hline
\end{tabular}

No tocante à mortalidade média observada (Tab. 4), os diferentes níveis de tiamulin empregados simultaneamente com salinomicina não afetaram a mesma. As aves que receberam $40 \mathrm{ppm}$ de tiamulin na ração foram as que apresentaram maior viabilidade, enquanto o pior resultado foi observado entre aquelas que receberam $30 \mathrm{ppm}$. Todavia. não foram detecladas diferenças significativas entre os tratamentos.

TABELA 4

Mortalidade observada no experimento. Pirassununga - SP. 28/11/89 a 16/01/90.

\begin{tabular}{ccc}
\hline Tratamentos & Mortalidade (1-49 dias $)$ & $\%$ \\
\hline A & $14 / 216$ & 6.48 \\
B & $12 / 216$ & 5.55 \\
C & $16 / 216$ & 7.40 \\
D & $21 / 216$ & 10.64 \\
E & $7 / 216$ & 3.24 \\
F & $15 / 216$ & 6.94 \\
\hline
\end{tabular}

Quanto aos resultados do exame de lesões, feito aos 45 dias, verificou-se que. para todos os tratamentos, não ocorreram lesões nos sacos aéreos e as amostras de soro, usadas em soroaglutinação rápida para Mycoplasma gallissepricum, apresentaram resultados negativos. 


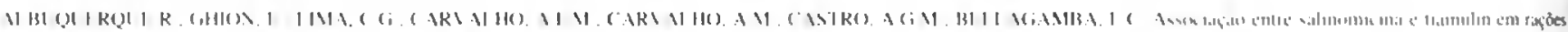

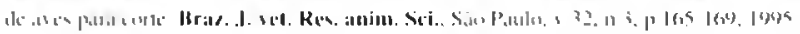

\section{CONCIUSOES}

Nas condiçós ém gue o presente experimento foi realizado.

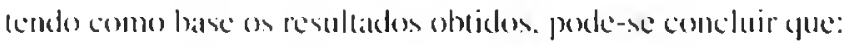

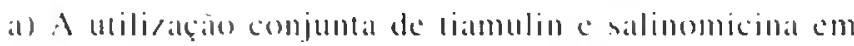

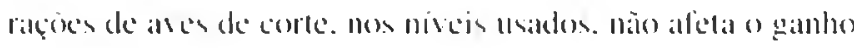
(2)ו p'oro.

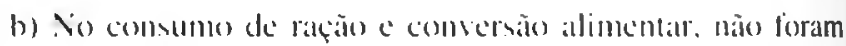
obseradas alteraçoós quando se adicionou balinomicina com on sem liamulin.

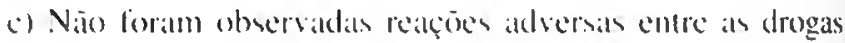
utilizalats nos diferenter tratamentor. Arsim. em frangos de

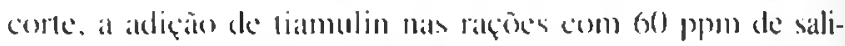
nomicina, nim apresental cleitor loxicos.

\title{
SIMNK)
}

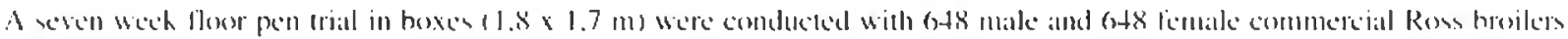

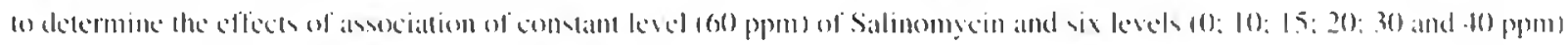

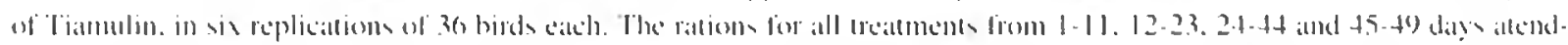

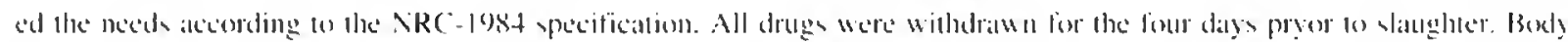

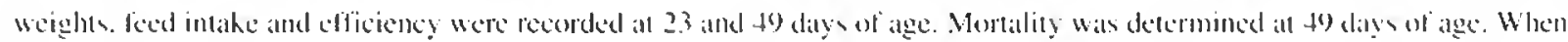

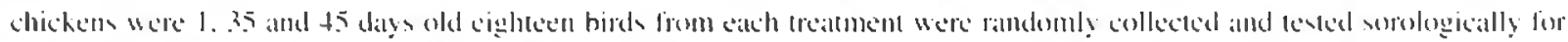

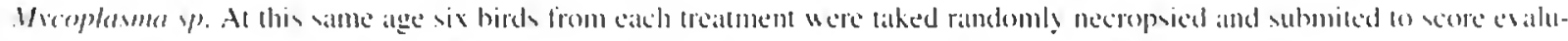

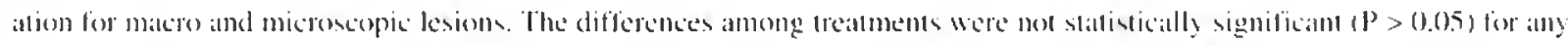

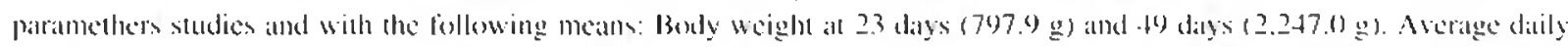

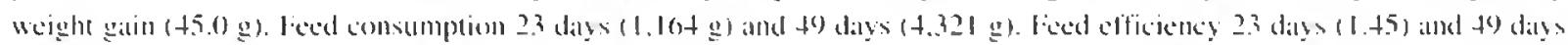

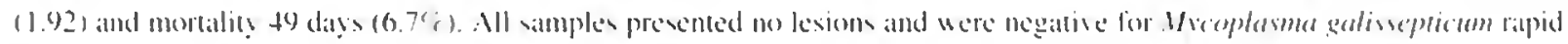

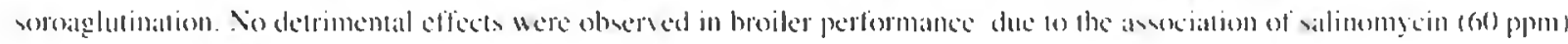
and liamulin (10 a 40 ppms).
\end{abstract}

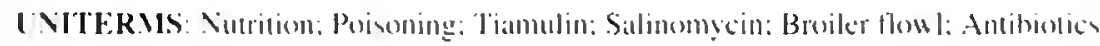

\section{REFERÊNCIAS BIBIIOGRÁFICAS}

1-B3OR(H. 1).C.S. Toxicity of the ionophores. The Veterinary Record, s.114. p.27\%, 1984.

2-DANHORTH, H.D.: R(HF: M.D.: RHII). WI.M.: J(MINSON. J. Anticoceidial activity of salinomycin in flow pell expreriments will broikers. Poultry science, v.56. p.9.3.38. 1977.

3-1)A.VFORTH. H.D.: RLFF. M.I): REID, W.M.: MII.I.FR. R.I.. Anticoccidial atctivity of salimonycin in hatlery raised broiler chicks. Poultry Science. 1.56. p.926-32. 1977.

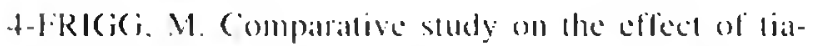
mulin in chichens receising lead containing ciller lanilocid or moncosin. Kesearch ('uisclosure. 1, 177, p.24-6. 1979.

5-FRIGG, M.: BROZ. J.: WEBER, (i. Compalibility studies of ionophore anticocidials with various ancibiotics and chemotherapeutic in broiler chicks. Archiv für Gieffugelkunde, $1 .+7,0.5, p, 21.3-20.1983$.
6-HORROX. N.I: Monemsin tiamulin interaction rivk 10 poultry. The Veterinary Record. 4.106, p.278. 1980.

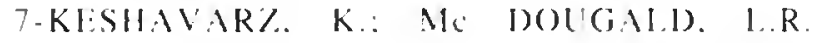
Anticocidial druger: growh and performance depressing

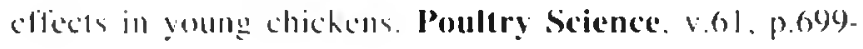
705. 1982

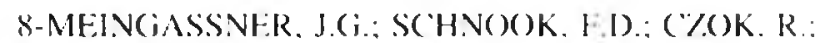
MIE:l'H. H. Finhancement of the anticoccidial aldivily of polyether antibiolice in chickems by liamulin. Poultr? science. $1.58,0.308-1.3 .1079$.

9-MICAAKI, T.T: CIIAPII:L I.R.: BABCOC'K. W.I: Anticocidial ellicaley of a new polyedher antibiotic. salinomycin. in comparison to momensin and lasalocid in batters trials. Poultry Science. 1. 58. p.1192-6. 1979.

I()-MORRISON, W.I) : FIRGUSON, A.E.: IIEASON. S. Efficacy of salinomycin and sencrol agaimst various species of Eimerial and clfect on chick performance. Poultry Science. v. 58 , p.1160-6, 1979. 


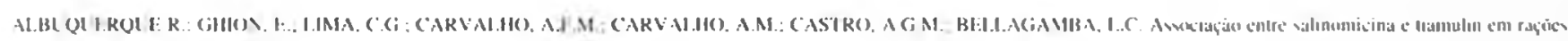

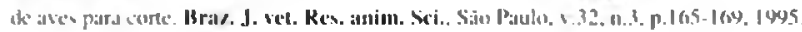

I I-NATIONAL, RESEARCH COUNCIL. Nutrient requirements of domestic animals. Washington. National Academy of Sciences, 1984.

12-S.VEDP:COR. G.W.: (O)(HRAN. W.D. Statistical metheds. 7. ed. Ames. The lowa State Lniversity Press. 1980.

1.3-SCY.A.VCIER. A.J. Toksicitet of jonophore-polyether antihiotika. Dansk Veterinar Tidsskrift, v.72, p.744-51. 1989.

I4-WEISMAN, Y.: SHLOSBERG, A.: EGYYI), M.N. Acute poisoning in turkeys cause by incompatibility of mon- ensin and tiamulin. Veterinary Rescarch Communications, v.4. p.231-5, 1980).

I.5-YVORE, P.: RAYNOUD, J.P.; COHAN, L.: NACIRI. M. Evoluation of the cfficacy of salinomycin in the control of coccidiosis in chicks. Poultry Science, v.59, p.2412-6. 1980. 